\title{
An Unusual Cause of Recurrent Apnea in an Infant: Eyes Say It All
}

\author{
Fajrudheen Mohamed $^{1} \cdot$ Bhagabat Nayak $^{2} \cdot$ Amit Kumar Satapathy $^{1}$
}

Received: 4 October 2021 / Accepted: 10 December 2021 / Published online: 31 January 2022

(c) Dr. K C Chaudhuri Foundation 2022

To the Editor: Recurrent apnea is a common presentation of central nervous system (CNS) or respiratory pathologies in infants. We came across an infant presenting with recurrent apnea and hypotonia, but with an unexpected and preventable cause, brimonidine eye drop-induced apnea and hypotonia.

A 6-mo-old boy was referred with a history of recurrent episodes of apnea for $15 \mathrm{~d}$. He was first born out of nonconsanguineous marriage without any perinatal adverse event. His developmental milestones were normal for age. Parents noticed episodes of reduced activity and decreased respiratory effort leading to desaturation, requiring bag and mask ventilation for the last $15 \mathrm{~d}$. It was not associated with seizure. There was no evidence of metabolic derangement on evaluation. He was started on antiepileptics in view of possibility of seizure. Electroencephalogram and magnetic resonance imaging of brain did not show any abnormality. On detailed history, parents gave the history of the child being diagnosed with congenital glaucoma of right eye, on the same day the episodes started. He was started on brimonidine eye drops, after which, episodes of apnea were noticed. So, possibility of brimonidine-induced CNS depression was kept and the drug was stopped, following which, there was no recurrence of apnea episodes. Alternate medication for glaucoma was started and he was planned for surgery.

Brimonidine is an alpha 2 agonist which crosses blood brain barrier and causes CNS depression [1]. Literature review showed that brimonidine causes CNS depression leading to hypotonia and apnea in infants and young children $[2,3]$. Other adverse effects include hypotension, miosis, bradycardia, hypothermia and hyporeflexia [4]. Our index case had recurrent apnea with hypotonia secondary to the topical brimonidine. Pediatrician should be aware of this potentially lethal adverse effect of brimonidine in infants.

\section{Declarations}

Conflict of Interest None.

\section{References}

1. Enyedi LB, Freedman SF. Safety and efficacy of brimonidine in children with glaucoma. J AAPOS. 2001;5:281-4.

2. Ghaffari Z, Zakariaei Z, Ghazaeian M, et al. Adverse effects of brimonidine eye drop in children: a case series. J Clin Pharm Ther. 2021;46:1469-72.

3. Prok L, Hall D. A 24-day old with episodic lethargy, hypotonia, and apnea: the eyes have it. Curr Opin Pediatr. 2003;15:226-8.

4. Rangan C, Everson G, Cantrell FL. Central alpha-2 adrenergic eye drops: case series of 3 pediatric systemic poisonings. Pediatr Emerg Care. 2008;24:167-9.

Publisher's Note Springer Nature remains neutral with regard to jurisdictional claims in published maps and institutional affiliations.

Amit Kumar Satapathy

amitkumar.satapathy@yahoo.co.in;

ped_amit@aiimsbhubaneswar.edu.in

1 Department of Pediatrics, All India Institute of Medical Sciences, Bhubaneswar, Odisha 751019, India

2 Department of Ophthalmology, All India Institute of Medical Sciences, Bhubaneswar, Odisha, India 